\title{
The Use of Virtual Reality in A Chemistry Lab and Its Impact on Students' Self- Efficacy, Interest, Self-Concept and Laboratory Anxiety
}

\author{
Almer Gungor ${ }^{1 *}$ (D) Denise Kool $^{2}$ (D), May Lee ${ }^{2}$ (D) Lucy Avraamidou $^{2 *}$ (D) Niek Eisink $^{2}$ (D), \\ Bauke Albada ${ }^{3}$ (D), Koos van der Kolk ${ }^{4}$ (D) , Moniek Tromp ${ }^{2}$ (D) Johannes Hendrik Bitter ${ }^{3}$ (D) \\ ${ }^{1}$ Odisee University of Applied Sciences, BELGIUM \\ 2 University of Groningen, NETHERLANDS \\ ${ }^{3}$ Wageningen University, NETHERLANDS \\ ${ }^{4}$ Labbuddy, NETHERLANDS
}

Received 19 December 2021 - Accepted 10 February 2022

\begin{abstract}
The purpose of this study was to evaluate the impact of virtual reality on undergraduate students' self-efficacy, self-concept, interest, and laboratory anxiety in an introductory chemistry course. We used a mixed-methods approach to improve our understanding of how these factors mediate student learning. The findings showed that (i) the use of the virtual reality application had an overall positive impact on students' self-efficacy, self-concept, interest, and anxiety; and (ii) students who expressed some anxiety about doing the lab prior to the course reported the use of the virtual reality application decreased their levels of anxiety at the end of the lab. The implications of these findings speak to the potential value of the use of virtual reality applications in higher education and especially in situations when distance learning is the only option as well as in situations where the costs of real laboratories cannot be afforded.
\end{abstract}

Keywords: virtual reality, chemistry, lab anxiety, self-efficacy

\section{INTRODUCTION}

Laboratory work, typically defined as learning experiences in which students interact with materials and equipment to examine scientific phenomena, has had a central role in chemistry education around the world since the $19^{\text {th }}$ century. As Hofstein and Hugerat (2021) argued, laboratory work does not only support the development of practical abilities (e.g., conducting an experiment) but can also enhance learners' interest and curiosity, foster creative thinking and problemsolving activity, and develop conceptual understanding. However, Dalgarmo et al. (2009) indicated that many students feel anxious about using unfamiliar and expensive equipment in chemistry courses with laboratories, which can lead to chemistry anxiety, or chemophobia (Dalgarno et al., 2009). Students may also express anxiety about participating in laboratories, which has been linked to poor course performance (Rummey et al, 2019). This anxiety may also hinder students' ability to recall information when they are evaluated, which results in low performance (Maloney et al., 2013).

One approach to reducing student anxiety with respect to participating in laboratories is by supporting the development of their self-efficacy (Chan et al., 2021). Self-efficacy refers to individuals' beliefs in their ability to accomplish specific tasks (Bandura, 1997) and can sustain students' efforts toward achieving academic successes (Vuong et al., 2010). High self-efficacy has been related to success in physics (Cavallo et al., 2004; Kost et al., 2009; Shaw, 2004), mathematics (Lent et al., 1984), and chemistry (Zhou et al., 2019). For example, Zhou et al. (2019) found that students who scored in the top tenth percentile on a survey for self-efficacy in organic chemistry tended to engage with the online organic chemistry course more frequently and consistently than students who scored in the bottom tenth percentile on the survey. Self-efficacy in chemistry serves at the heart

(c) 2022 by the authors; licensee Modestum. This article is an open access article distributed under the terms and conditions of the Creative Commons Attribution License (http://creativecommons.org/licenses/by/4.0/). 


\section{Contribution to the literature}

- The purpose of this study was to evaluate the impact of a virtual reality application on undergraduate students' self-efficacy, self-concept, interest, and anxiety in an introductory chemistry laboratory course; previous studies examined these factors independently.

- This study examines the impact of a virtual reality application on factors directly associated with learning instead of its usability in education.

- In contrast to previous studies showing that hands-on laboratories cannot be replaced with virtual alternatives, this study suggests that the use of virtual reality applications may bolster undergraduate students' self-efficacy, self-concept, and interest in addition to reducing their anxiety about chemistry laboratories.

of the account of this study as a construct that influences chemistry learning and retention.

Self-efficacy is also closely intertwined with selfconcept, interest, and anxiety. Self-concept refers to individuals' perceptions regarding themselves (Marsh, 1990). Interest is the "focused attention and affective reaction" that students acquire by participating in class (Hidi, 1990). Lastly, anxiety refers to a feeling of nervousness or worry about something (Sinclair, 1993); in this case, laboratory anxiety typically refers to the fear of failure from past negative learning experiences. These factors, as existing literature showcases, intertwine and impact student learning.

The recent COVID-19 pandemic has spurred educators into using online education, distance learning, and virtual reality applications. As of late March 2020, over 850 million students and youth-roughly half of the world's student population-had to stay away from schools and universities due to the COVID-19 pandemic (UNESCO, 2020). Many higher education institutions, including the one that defined the context of this study, utilized videoconferencing and learning management systems to offer online education. However, Chan et al. (2021) argued online education should be immersive experiences for learners - such as those provided by virtual reality (VR) and augmented reality (AR) applications. Although the use of VR and AR applications have increased in the past few years, only a small number of studies have evaluated their effectiveness and use in university settings (Radianti et al., 2019; Wohlgenannt et al., 2019). For example, Radianti et al. (2019) indicated the evaluation of educational VR applications has primarily focused on its usability as a technological tool, and with respect to pilot projects and developmental work, rather than its impact on student learning. Likewise, immersive VR has mostly been used as part of pilot projects and development work rather than being applied regularly in actual teaching and learning at university level.

\section{Purpose of the Study}

For the purpose of this study, we evaluated a virtual reality application with respect to its impact on specific factors that mediate student learning: self-efficacy, self- concept, interest, and anxiety. We adopted a mixedmethods approach to data collection and analysis, which included a group of university students' responses to a pre- and post-questionnaire alongside a set of interviews. We used these four interrelated constructs, instead of focusing on self-efficacy alone, to obtain a more comprehensive understanding of the factors that mediate student learning. The research questions guiding our study were:

1. How do students' self-efficacy, self-concept, interest and anxiety change after using a VR application in the context of a university chemistry lab?

2. What are students' perceptions about the use of a virtual application in a chemistry lab?

\section{LITERATURE REVIEW}

Recent studies showed that virtual pre-laboratory preparations (e.g., discussions and interactive simulations) impact students' laboratory experiences in mostly positive ways (Chaytor et al., 2017; Kapici et al., 2020; Makransky et al., 2020; Sarmouk et al., 2019). Chaytor et al. (2017) found that undergraduate students who followed a pre-laboratory preparation course felt more prepared for the lab and perceived the course activities as very useful. This is especially important in laboratory courses. In chemistry laboratories, many students feel anxious about using unfamiliar and expensive equipment in an unfamiliar environment which leads to chemistry anxiety or chemophobia (Dalgarno et al., 2009). In the past few years, we have witnessed more and more studies examining the impact of online activities as part of such pre-laboratory preparation courses. In the next few paragraphs, we synthesize the findings of these studies.

Sarmouk et al. (2019) carried out an experimental study with second year pharmacy students designing and implementing an online learning resource. Their sample consisted of 137 students enrolled in the two sections of the course in the UK. The pre-laboratory online learning resource consisted of various components including the experimental demonstrations (videos and visual cues), underlying theory, safety information, a quiz, and the traditional laboratory script. 
Students in the experimental group could access this online learning resource two weeks prior to their laboratory class via the virtual learning environment Blackboard $\AA$, while the students in the control group could access the "traditional script and underlying theory". The researchers evaluated the effectiveness of the online learning resource by using various measures. To assess student engagement, they used the online tracking metrics on the Blackboard $\AA$. To evaluate the quality of the experimental practice, demonstrators/technicians recorded the number of student mistakes during the laboratory sessions. To evaluate the usefulness of the online resource, students completed an online survey. Their results revealed that after the online pre-laboratory preparation, the students were able to use the lab equipment with less support and anxiety, stating that they were more confident in the laboratory. The students in the experimental group also asked for less guidance and made fewer practical mistakes compared to the control group. Summing up, the findings of this study showed that the online prelaboratory preparation with varying learning activities improved the students' laboratory experiences by enhancing their preparedness, engagement, confidence, efficiency, and performance.

Makransky et al. (2020) found the interest and selfefficacy of middle and high school students increased when immersive virtual reality laboratory simulations were used in their science courses. Other studies found that virtual pre-laboratory preparations resulted nonsignificant changes when compared to hands-on preparations (Dalgarno et al., 2009, 2012; Kapici et al., 2020). Similarly, Kapici et al. (2020) found that seventh graders using the virtual laboratory had higher gain on attitude questionnaire (including self-concept) from pretest to posttest than those who completed the handson laboratory. However, the improvement in students' attitudes (e.g., self-concept) towards science courses and laboratories was not statistically different among the three groups (i.e., hands-on, virtual, or combination).

In a study that aimed to compare the learning of students who engaged with a virtual laboratory lab and those who participated in a physical laboratory, Dalgarno et al. (2009) provided students a CD-ROM containing a virtual chemistry laboratory-a simulated $3 \mathrm{D}$ representation of the laboratory-to help students become familiarized with the laboratory environment and equipment before the laboratory session. Their sample consisted of 22 students, with half of the students in the virtual laboratory group while the other half in the real laboratory group. The students in the experimental group explored the virtual laboratory, and the control group took a tour of the real laboratory. Both tours were 40 minutes. The experimental group received instructions on a page as a reference during the virtual laboratory and students in this group were asked to learn the layout of the laboratory, locate various equipment such as beakers, pipettes, and items of larger equipment, and to learn their structure. They were asked to record the items they were able to locate on a list provided by the researchers and to take notes that might be helpful to remember the layout of the laboratory. The students in the control group visited some locations in the laboratory in groups of 3-6. After the equipment was introduced to them and their locations in the laboratory were shown, the participants were asked to explore the equipment. Following that, the ability of the participants to recall the laboratory layout and familiarity with the equipment was tested. This was done in order to check if learning in the virtual laboratory tour was similar to learning in a real laboratory tour.

The participants in both groups completed several tests including an apparatus identification test, a laboratory layout test, and an apparatus location test. Even though their sample size was small, they conducted a factorial analysis of variance (ANOVA) with group and gender as factors and the test scores as dependent variables. Although the mean scores for the real laboratory group were slightly higher than those for the virtual laboratory group, the differences were not statistically significant for apparatus identification and laboratory layout tests. However, they reported a statistically significant difference in favor of the real laboratory group only for the apparatus location test. The researchers concluded that the virtual laboratory is almost as effective as the real laboratory in gaining familiarity with the laboratory environment and equipment.

Moreover, the questionnaire and interview data supported the usefulness of the virtual laboratory that the students stated that they would recommend its further use. However, many students stated that lack of familiarity was not a main source of anxiety in the laboratory, and thus the virtual laboratory did not have an influence on their laboratory experience. They stated that the main source of their anxiety in the lab was not being able to apply mathematical techniques and chemistry concepts during the laboratory sessions. In Dalgarno et al.'s (2009) study, a sample of 22 students were sorted into a virtual or real laboratory group. Both groups were provided explicit instructions regarding tasks to complete (e.g., locating beakers and pipettes) as they explored the virtual or real laboratory. Assessments on these tasks showed that students in the real laboratory group had slightly higher scores, though the difference was not statistically significant. Most of the students stated the virtual laboratory did not influence their lab experience because lack of familiarity was not a source of anxiety for them; instead, they identified the application of mathematical techniques and chemistry concepts during lab as their main sources of anxiety.

Following these findings, the researchers listed some other benefits of the pre-laboratory preparation, in addition to students being more relaxed and comfortable 
in the laboratory: (i) less time was spent towards searching for lab equipment; (ii) improved safety; (iii) supporting students in building and using laboratory equipment; and (iv) more attention was provided to conceptual understanding given the familiarity with the experimental procedures.

In a follow-up study, Dalgarno et al. (2012) found the virtual chemistry laboratory had little effect on students' confidence in the first practical session; students using the virtual laboratory reported that it enhanced their confidence and reduced their anxiety but did not help them assemble and operate equipment.

Building upon these findings, in this study we aimed at gaining a more comprehensive understanding of the impact of a virtual reality application on four different factors associated with learning and also through the use of a mixed-methods approach that provide both an exploratory and explanatory understanding.

\section{METHODS}

\section{Context and Participants}

The participants were 17 second- and third-year pharmacy students who were enrolled in a three-week introductory organic chemistry practical course at a northern European university in spring of 2021. The students followed theoretical lectures during the first two weeks and completed laboratory experiments in the third week. The course started with a practical on using the VR application.

The app was created using the Unreal 4 engine (https://www.unrealengine.com). The VR hardware used is the Oculus Quest ${ }^{\mathrm{TM}}$ (https:/ / www.oculus.com)see Figure 1 and Figure 2. We provide an overview of the three design aspects of the application: (i) technical, (ii) design, and (iii) educational. In terms of technical characteristics, we wanted to design an app that runs on Oculus Quest ${ }^{\mathrm{TM}}$-devices, which do not require to be connected to external computers. In addition, the screen in VR should be casted to an external monitor using Google Chromecast, allowing peer students and teachers to see what the student performing the experiment is doing. In terms of design, we aimed at designing an app that could be usable for various organic chemistry laboratories at different educational institutes. In addition, we wanted the app to be as 'content-agnostic' as possible, allowing for usage in various laboratory courses. The organic chemistry content can be (more easily) delivered to students via other media. In terms of education, our goal was to make the real organic chemistry laboratory practical more efficient and effective, by making students acquainted with building an organic chemistry reaction setup inside a fume hood and by reducing 'laboratory anxiety'.

We chose this VR application for two reasons. First, we wanted the application to run on Oculus Quest ${ }^{\mathrm{TM}}$ -

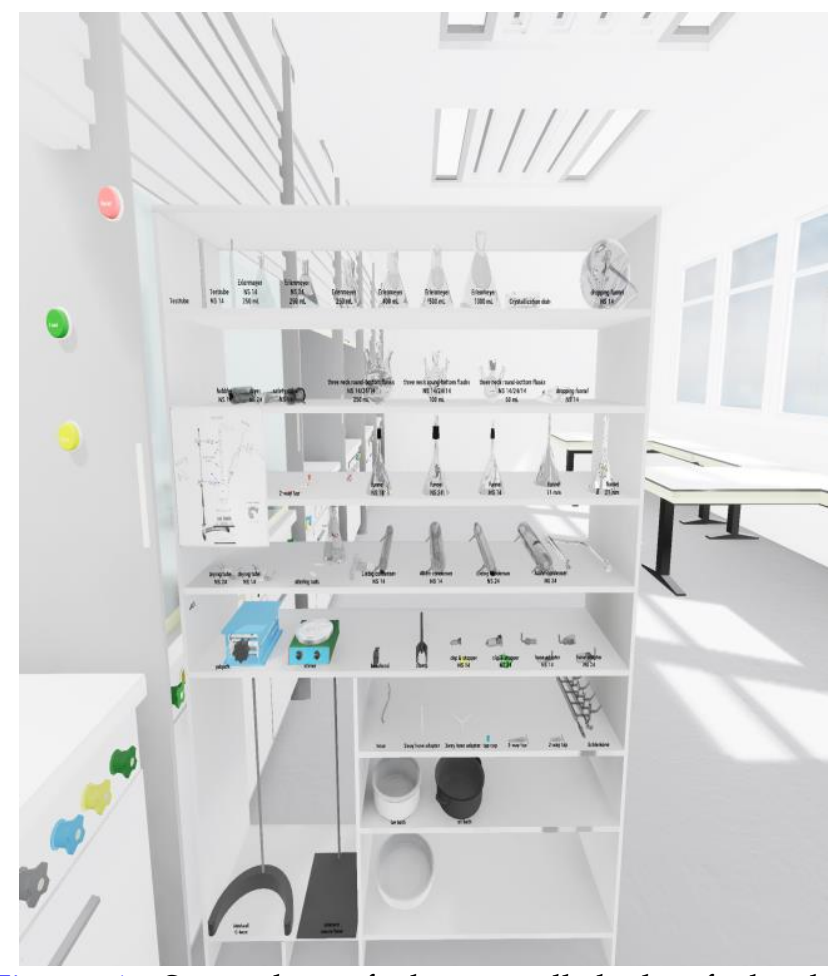

Figure 1. Screenshot of the overall look of the lab environment in the VR application

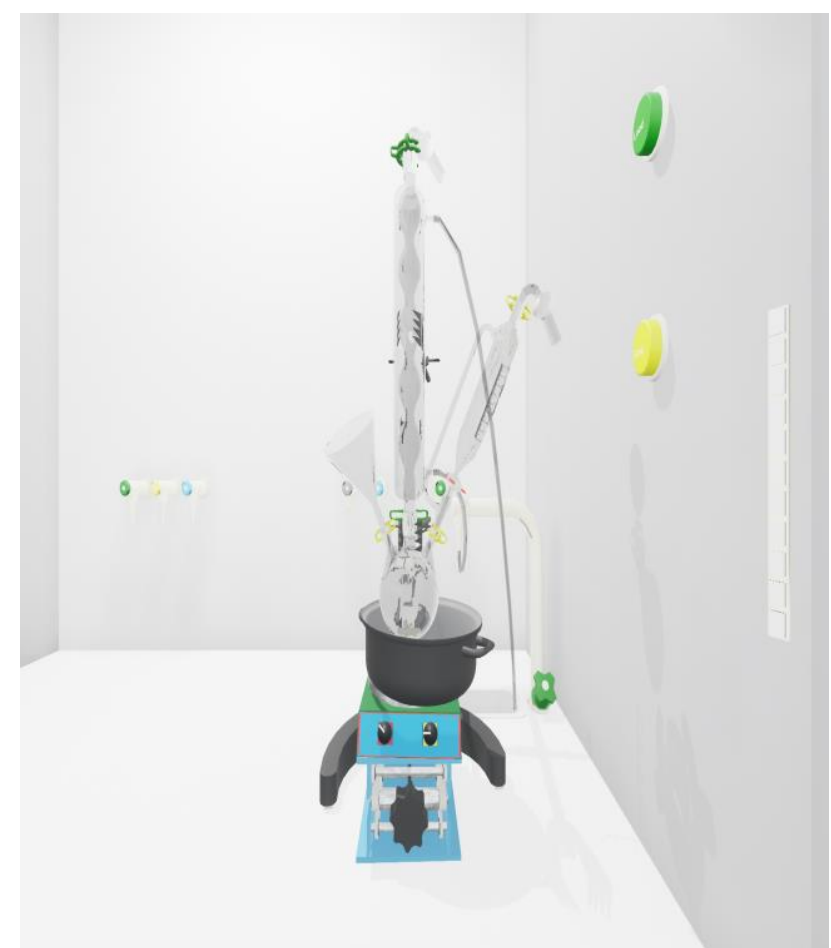

Figure 2. Screenshot of an experimental set up in the VR application

devices that can be screen-casted to external monitors, which allowed students and instructors to see experiments performed by any student. Second, we wanted the application to help reduce (any) laboratory anxiety students may have with respect to setting up organic chemistry reactions under a fume hood. 


\section{Instruments}

To gain a comprehensive understanding of the students' experiences with the VR application, we asked the participants to respond to a questionnaire and participate in semi-structured focus group interviews. The questionnaire was adapted from the affective characteristics scale (Gungor et al., 2007), which addresses 7 subscales. We focused on four subscales from this questionnaire (self-efficacy, self-concept, interest, and anxiety). In previous studies, this questionnaire was administered to: (i) predict students' physics achievement (e.g., Abak, 2003), (ii) study gender differences in physics courses (Gungor \& Eryilmaz, 2006), (iii) measure the impact of various context-based interventions in physics courses (e.g., Pesman \& Ozdemir, 2012), and (iv) explore the effectiveness of context-based instruction on students' affective characteristics in chemistry (Sunar, 2013).

The items on the questionnaire had already been translated and validated in the language of the participants (Gungor \& De Cock, 2021), which was then adapted to address the organic chemistry laboratory context for this study. A team of experts (a professor in education and learning sciences, an education expert with a doctoral degree in chemistry education, a master student with specialization in chemistry education) reviewed the items to ensure the adaptations were coherent.

Before students used the VR application (pre-study), 17 students completed the questionnaire and three groups (six students) were interviewed about their (i) expectations from the course, (ii) views on VR in general, and (iii) motivation.

After the last experiment was completed (poststudy), 14 students completed the same questionnaire (one student left the course, two students did not participate) and four groups (ten students) were interviewed about their experiences with the course, views on using the VR application, and its impact on their learning.

Semi-structured interviews were conducted in groups between two and three students for the purpose of triangulation but also for gaining a more in-depth understanding about the students' experiences. Six students were interviewed before the VR experience about their expectations of the course, views on VR, and motivation. Ten students were interviewed after the course and responded to questions related to their experiences as students in the course, their views on VR, and their motivation as learners.

For the questionnaire, the responses to each subscale were averaged, with the minimum and maximum scores noted. For the focus group interviews, the responses were characterized as expressing positive, neutral, or negative perspectives.
Table 1. Descriptive analysis of pretest \& posttest

\begin{tabular}{lcccccccc}
\hline & \multicolumn{3}{c}{ Self-efficacy } & \multicolumn{2}{c}{ Self-concept } & \multicolumn{2}{c}{ Interest } & \multicolumn{2}{c}{ Anxiety } \\
\hline & Pre & Post & Pre & Post & Pre & Post & Pre & Post \\
\hline $\mathrm{N}$ & 17 & 14 & 17 & 14 & 17 & 14 & 17 & 13 \\
Min & 3.00 & 3.00 & 3.00 & 3.00 & 2.75 & 3.25 & 1.00 & 1.00 \\
Max & 4.40 & 5.00 & 4.40 & 4.80 & 5.00 & 5.00 & 4.20 & 3.00 \\
Mean & 3.79 & 4.06 & 3.72 & 3.84 & 3.74 & 4.09 & 2.53 & 1.78 \\
SD & .35 & .50 & .34 & .51 & .56 & .62 & .96 & .67 \\
\hline
\end{tabular}

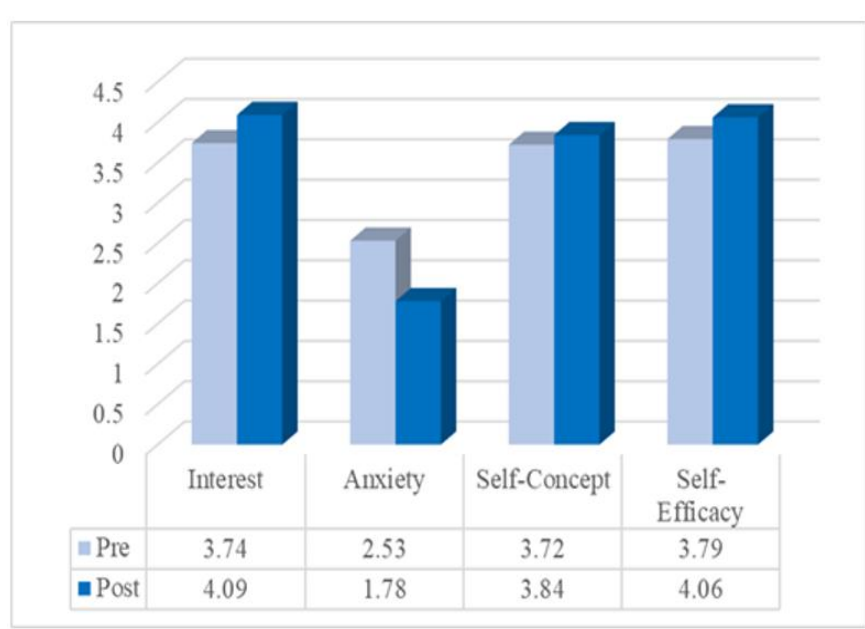

Figure 3. Comparison of subscales of the questionnaire pretest \& posttest

\section{FINDINGS}

Overall, the findings showed that the incorporation of the VR application in the course had a positive impact on students' interest, anxiety, self-concept, and selfefficacy. Students reported an increase in positive association confidence in the lab, and a decrease in laboratory anxiety. The descriptive statistics for the subscales of the questionnaire are presented in Table 1. The pre-study data of the three students who did not complete the questionnaire in the post-study were not excluded in the descriptive statistics due to the small sample size. Without these students' data, the means of the pretest scores for self-concept decreased to 3.65. This difference in scores suggest that the three students who did not complete the post-study questionnaire started with higher self-concept compared to the group; however, their scores with respect to self-efficacy, interest, and anxiety were similar to the scores of the other students. As shown in Figure 3, students' interest, self-concept and self-efficacy increased slightly, while their anxiety decreased comparatively more, after the last laboratory. Students started and finished the lab with a medium-high level interest, self-concept, and selfefficacy, ranging from 3.72 to 3.79 for the pretest and from 3.84 to 4.09 for the posttest. The mean score for their anxiety was 2.53 (slightly lower than the neutral level), meaning that they had a medium level anxiety at the beginning and ended with a lower mean score (1.78) compared to the initial level. 
In the following sections, we address patterns we found amongst the students' responses about their preand post-course impressions about the lab (e.g., lab anxiety, use of virtual reality).

\section{Pre-Course Impressions}

Prior to the start of the course, the students' contrasting impressions of the lab were informed by various sources. For instance, one of S1's personal impressions about the lab was to associate it with "more dirty lab coats" but also noted that "really everyone I hear about it says, the organic chemistry lab that's really fun." S3 expected the course "will be okay" because "I always notice that if I understand it well, if I'm well prepared, then I expect it to turn out all right." S3's response suggests high self-efficacy. S2 also heard "positive signals" about the lab and expressed high expectations for it, but still indicated not knowing what to expect. Without addressing "lab anxiety," S2 indicated experiencing stress about the course due to not having completed "a practical course in a long time" and concerns about keeping pace with other students in the class:

I think too much. I think so much, and instead of enjoying the practicum, I start thinking about the time and then I feel even more stress. Then it doesn't go as well as when I don't experience pressure. Someone is around you all the time. Someone has to meet a certain pace. Whereas my pace is first to think carefully, to look carefully at each action to see if everything is correct. But you still have to take the pace of the average and some work faster than others (S2).

S2's response suggests low self-efficacy. In contrast to $\mathrm{S} 1$ and S2, S6 presented impressions that focused on the consequences of making mistakes in the lab, stating "You are not allowed to make mistakes. Three strikes and you have to bring cake when you break something." Responses from S6 suggest these impressions may be a source of stress or lab anxiety.

With respect to VR, two students had no previous experience (S1 and S5) and two expressed they had previous VR experience (S2 and S4). While S5 expressed excitement for the opportunity to try out the VR glasses, S1 did not express any positive or negative anticipation. Of those who used VR previously, S2 noted the mindbody disconnect between VR and the real world when wearing the VR glasses and advised not wearing the glasses too long, otherwise users may experience headaches. Similarly, S4 described the visceral sensations experienced from putting the VR glasses on, noting the strangeness of the experience because "you're in a different room, so your sensory organs can get a bit confused, but it is really fun to emerge yourself in a wholly different room like that." These prior experiences likely influenced the students' current perceptions about using VR in the lab.

Three students expressed ambivalence about the usefulness of VR with respect to the practical or the profession (S3, S4, and S6). S1 and S2 indicated VR could help people "mentally prepare" for an upcoming practical. S5 suggested VR could be useful by taking away some of the nervousness associated with lab work, but S6 (acknowledging not knowing what to expect from VR) disagreed because doing VR was not comparable to doing "real work."

Four students said they did not know about the impact of VR with respect to their motivation for the practical because it depends on how the VR would be used. For instance, S3 expressed high self-motivation for the course without using the VR but acknowledged it might increase motivation for those who felt isolated due to pandemic. S5 also indicated that VR would not affect self-motivation for the practical and noted VR could create an artificial separation between VR and non-VR days: "I feel like in my mind, I'm separating today and other lab days like they're not connected at all. So, I'm not sure if it'll affect my motivation for the rest of the course." When comparing the amount of learning with and without VR, S6 indicated there was no difference, a sentiment which S4 and S5 echoed.

\section{Post-Course Impressions}

Three students (S7, S9, and S12) expressed positive impressions about the course that exceeded their initial expectations-such as, "I think it was even more fun than expected" (S9) and "It was better than I thought" (S12). S12 noted the lab was not stressful because the responsibility of doing the lab correctly was shared by the teaching assistant. In contrast, S16 indicated experiencing nervousness attributed to a lack of knowing what to expect from the lab because "the preparation is always a bit of a grey area for me."

Four students acknowledged the use of VR in the lab was "nice" and "interesting" but not necessarily essential or helpful for educational purposes. While S13 and S16 indicated they did not learn much from using VR in the lab, they acknowledged that VR may "help if someone has never set up a facility like that" (S13) and could be "a kind of steppingstone to the real thing and that you did meet physically one more time to ask questions about the lab" (S16). S12 indicated that using VR requires more time "because then the teaching assistant has to teach you how to build the setup and help us with that" and critiqued the authenticity of the VR experience, noting that "you can throw the glassware on the ground and it disappears [in VR]. That doesn't happen [in real life] of course." S15 shared the same critique by noting that "in VR you could put the screen down, and your hands could go through the glass," which may be problematic because VR contributes little 
to the building of safety habits ("...we've never worked in a fume hood. It's not hard either, but you really have to have that discipline to keep your hands in the fume hood and work with the substance there. You might be able to do that a little bit with that VR.").

Two students indicated VR reduced some of their anxiety about lab, citing its usefulness in preparing for lab. S11 indicated using VR decreased some anxiety because a person can "already start with part of the experiment so you already know what you get to do the next day." Similarly, S14 addressed how using VR provides an opportunity to "indeed practice first and then you are prepared for the next day."

Three students expressed ambivalence or experiencing a general lack of lab anxiety. S10 expressed that the VR "was fun, like a video game" but did not relate to lab anxiety whereas S16 expressed "I don't really have anxiety about being in the lab or working with chemicals." S8 perceived that VR helped more with learning the various glassware and how to assemble them rather than with lab safety, which S8 seemed to associate with lab anxiety. Two students stated VR did not help (much) with reducing lab anxiety. S7 attributed lab anxiety to writing a 30-page lab report late the night before needing to be present at the lab early the next morning rather than with concerns of lab safety. S15 stated that VR did not help reduce anxiety because it presents a false sense of confidence. S15 explained how people might think they are prepared to do everything well after using VR, but it's more stressful because they are not guided anymore when performing the physical lab.

Overall, these findings indicate that most students perceived the VR as a positive experience, while only a couple of students expressed ambivalence-they were not able to connect the experience to perceived learning, and two stated that VR provides a sense of false confidence. This might be explained by the lack of time for reflection on learning given that the interviews were held right after the course as well as the fact that the VR experience served as only one component of the course instead of being used throughout the duration of the course.

\section{CONCLUSIONS AND IMPLICATIONS}

Overall, the findings showed that students started the lab with high interest, in addition to high self-concept and self-efficacy, that were sustained over the course of the lab. Moreover, the findings revealed that students started with relatively lower levels (medium level) of lab anxiety, which decreased by the end of the week. Since we were only able to measure these variables before and just after the course was complete, one can interpret these changes as a result of the combination of using VR as a pre-laboratory learning tool with the traditional lab sessions. Our findings are in line with previous research in which preparation courses in various formats were found to foster motivation in chemistry laboratories (Chaytor et al., 2017; Dori et al., 2003, Zhou et al., 2020). Despite earlier empirical evidence showing that handson laboratories cannot be replaced, this study adds to growing evidence pointing to the fact that virtual chemical laboratories are not only viable as effective complementary tool, but that they provide better results in learning outcomes (Chan et al., 2021).

From a more practical perspective, virtual reality labs provide an effective tool for distance learning, especially in situations when distance learning is the only option as well as in situations where the costs of real laboratories cannot be afforded. In this sense, virtual reality technology has the potential to foster equity and inclusion by lifting up the barriers raised by physical distance and access to resources. Hence, we would argue that future research examines the potential of the use of virtual reality laboratories for purposes that go beyond teaching and learning and relate to issues related to equity and inclusion in education.

One limitation of this study is the non-matching responses between the pre-study and post-study questionnaires and interviews, which could have shown how individual students' responses changed with respect to their use of the VR application. However, the findings show that one week of intense organic chemistry laboratory preceded by a VR pre-laboratory intervention has the potential to foster self-efficacy beliefs and to decrease lab anxiety. Therefore, we would recommend further studies replicating this preliminary study with a larger sample size with an experimental design for the purpose of obtaining more generalizable results as well as an understanding of the changes with respect to individual students. Another limitation of the study is associated with the novelty effect given that virtual reality applications are not currently widely utilized in higher education. Hence, future studies should extent the duration of the virtual reality experience within a series of courses and carry out longitudinal investigations for the purpose of controlling for the novelty effect. In addition, it is likely that specific affordances of the Oculus Quest ${ }^{\mathrm{TM}}$. have contributed to these results in ways that other types of devices would not have. Hence, we recommend that future research compares and contrast the ways in which different VR applications impact students' self-efficacy, self-concept, interest, and anxiety for the purpose of better understanding the unique impact of each application.

Author contributions: All authors have sufficiently contributed to the study, and agreed with the results and conclusions.

Funding: The study was funded through a grant through the Open and Online education incentive scheme made available by the Dutch Ministry of Education, Culture and Science.

Declaration of interest: No conflict of interest is declared by authors. 


\section{REFERENCES}

Abak, A. (2003). Modeling the relationship between university students' selected affective characteristics and their physics achievement [Unpublished master's thesis, Middle East Technical University, Ankara, Turkey].

Agustian, H. Y., \& Seery, M. K., (2017). Reasserting the role of pre-laboratory activities in chemistry education: a proposed framework for their design. Chemistry Education Research and Practice, 18(4), 518532. https:/ / doi.org/10.1039/C7RP00140A

Bandura, A. (1986). Social foundations of thought and action: A social cognitive theory. Prentice Hall.

Benden, D. K., \& Lauermann, F. (2021). Students' motivational trajectories and academic success in math-intensive study programs: Why short-term motivational assessments matter. Journal of Educational Psychology. https://doi.org/10.1037/ edu0000708

Cavallo, A. M. L., Potter, W. H., \& Rozman, M. (2004). Gender differences in learning constructs, shifts in learning constructs, and their relationship to course achievement in a structured inquiry, yearlong college physics course for life science majors. School Science and Mathematics, 104(6), 288-300. https:// doi.org/10.1111/j.1949-8594.2004.tb18000.x

Chan, P., Van Gerven, T., Dubois, J.-L., \& Bernaerts, K. (2021). Virtual chemical laboratories: A systematic literature review of research, technologies and instructional design. Computers and Education Open, 2, 100053. https://doi.org/10.1016/j.caeo.2021. 100053

Chaytor, J. L., Al Mughalaq, M., \& Butler, H. (2017). Development and use of online prelaboratory activities in organic chemistry to improve students' laboratory experience. Journal of Chemical Education, 94(7), 859-866. https://doi.org/10.1021/acs. jchemed.6b00850

Dalgarno, B., Bishop, A. G., Adlong, W., \& Bedgood Jr, D. R. (2009). Effectiveness of a virtual laboratory as a preparatory resource for distance education chemistry students. Computers $\mathcal{E}$ Education, 53(3), 853-865. https://doi.org/10.1016/j.compedu.2009. 05.005

Dalgarno, B., Bishop, A. G., Bedgood Jr, D. R., \& Adlong, W. (2012). What factors contribute to students' confidence in chemistry laboratory sessions and does preparation in a virtual laboratory help? In Proceedings of the Australian Conference on Science and Mathematics Education (Vol. 10). https:/ / openjournals.library.usyd.edu.au/index.p hp/IISME/article/view/6490

Dori, Y. J., Barak, M., \& Adir, N. (2003). A Web-based chemistry course as a means to foster freshmen learning. Journal of Chemical Education, 80(9), 10841092. https:/ / doi.org/10.1021/ed080 p1084

Gungor, A. A., \& Eryilmaz, A. (2006). Gender differences in freshmen's physics related affective characteristics. GIREP Conference 2006: Modelling in Physics and Physics Education. Amsterdam, Netherlands.

https:/ / lirias.kuleuven.be/ retrieve/619328

Gungor, A. A., Eryilmaz, A., \& Fakioglu, T. (2007). The relationship of freshmen's physics achievement and their related affective characteristics. Journal of Research in Science Teaching,44(8), 1036-1056. https://doi.org/10.1002/tea.20200

Gungor, A., \& De Cock, M. (2021). Validity of the revised physics affective characteristics scale for Flemish pharmacy and biology majors. Physical Review Physics Education Research, 17(1), 114. https:/ / doi.org/10.1103/PhysRevPhysEducRe s.17.010132

Hidi, S. (1990). Interest and its contribution as a mental resource for learning. Review of Educational research, 60(4), 549-571. https:/ / doi.org/10.3102/003465430 60004549

Hofstein, A., \& Hugerat, M. (2021). Teaching and learning in the school chemistry laboratory. The Royal Society of Chemistry. https://doi.org/10.1039/978183916 4712-00001

Kapici, H. O., Akcay, H., \& de Jong, T. (2020). How do different laboratory environments influence students' attitudes toward science courses and laboratories? Journal of Research on Technology in Education, 52(4), 534-549. https:/ / doi.org/10.1080/ 15391523.2020.1750075

Kost, L. E., Pollock, S. J., \& Finkelstein, N. D. (2009). Unpacking gender differences in students' perceived experiences in introductory physics. AIP Conference Proceedings, 1179(1), 177-180. https://doi.org/10.1063/1.3266708

Lent, R. W., Brown, S. D., \& Gore Jr, P. A. (1997). Discriminant and predictive validity of academic self-concept, academic self-efficacy, and mathematics-specific self-efficacy. Journal of counseling psychology, 44(3), 307. https://doi.org/ 10.1037/0022-0167.44.3.307

Makransky, G., Petersen, G. B., \& Klingenberg, S. (2020). Can an immersive virtual reality simulation increase students' interest and career aspirations in science? British Journal of Educational Technology, 51(6), 2079-2097. https://doi.org/10.1111/bjet. 12954

Maloney, E. A., Schaeffer, M. W., \& Beilock, S. L. (2013). Mathematics anxiety and stereotype threat: Shared mechanisms, negative consequences and promising interventions. Research in Mathematics 
Education, 15(2), 115-128. https:/ / doi.org/10.1080/ 14794802.2013.797744

Marsh, H. W. (1990). A multidimensional, hierarchical model of self-concept: Theoretical and empirical justification. Educational Psychology Review, 2(2), 77172. https:/ / doi.org/10.1007/BF01322177

Pesman, H., \& Ozdemir, O. F. (2012). Approach-method interaction: The role of teaching method on the effect of context-based approach in physics instruction. International Journal of Science Education, 34(14), 2127-2145. https://doi.org/10.1080/ 09500693.2012.700530

Pogacnik, L., \& Cigic, B. (2006). How to motivate students to study before they enter the lab. Journal of Chemical Education, 83(7), 1094. https:/ / doi.org/ 10.1021/ed083p1094

Radianti, J., Majchrzak, T. A., Fromm, J., \& Wohlgenannt, I. (2019). Virtual reality applications for higher education: Design elements, lessons learned, and research agenda. Computers $\mathcal{E}$ Education, 147, 1-29.

Rummey, C., Clemons, T. D., \& Spagnoli, D. (2019). The impact of several demographic factors on chemistry laboratory anxiety and self-efficacy in students' first year of university. Student Success, 10(1), 87-99. https://go.gale.com/ps/i.do?p= AONE\&sw $=w \& i s s n=22050795 \& v=2.1 \& i t=r \& i d=G$ ALE\%7CA581622977\&sid=googleScholar\&linkacc ess $=$ abs

Sarmouk, C., Ingram, M. J., Read, C., Curdy, M. E., Spall, E., Farlow, A., Kristova, P., Quadir, A., Maatta, S., Stephens, J., Smith C., Baker, C., \& Patel, B. A. (2019). Pre-laboratory online learning resource improves preparedness and performance in pharmaceutical sciences practical classes. Innovations in Education and Teaching International, 57(4), 460-471. https://doi.org/10.1080/14703297. 2019.1604247

Shaw, K. A. (2004). The development of a physics selfefficacy instrument for use in the introductory classroom. AIP Conference Proceedings, 720, 137-140. https:/ / doi.org/10.1063/1.1807273
Sinclair, J. (Ed.). (1993). Collins COBUILD English language dictionary. Harper Collins Publishers.

Sunar, S. (2013). The effect of context-based instruction integrated with learning cycle model on students' achievement and retention related to states of matter subject [Unpublished master's thesis]. Middle East Technical University.

UNESCO. (2020, March 19). Half of world's student population not attending school: UNESCO launches global coalition to accelerate deployment of remote learning solutions. UNESCO. https:/ / en.unesco.org /news/half-worlds-student-population-notattending-school-unesco-launches-globalcoalition-accelerate

Vuong, M., Brown-Welty, S., \& Tracz, S. (2010). The effects of self-efficacy on academic success of firstgeneration college sophomore students. Journal of College Student Development, 51(1), 50-64. https:/ / doi.org/10.1353/csd.0.0109

Wohlgenannt, I., Fromm, J., Stieglitz, S., Radianti, J., \& Majchrzak, T. A. (2019). Virtual reality in higher education: Preliminary results from a DesignScience-Research project. In A. Siarheyeva, C. Barry, M. Lang, H. Linger, \& C. Schneider (Eds.), International Conference on Information Systems Development (ISD). ISEN Yncréa Méditerranée. https:/ / aisel.aisnet.org/isd2014/proceedings2019 /NewMedia/5

Zhou, N., Fischer, C., Rodriguez, F., Warschauer, M., \& King, S. (2020). Exploring how enrolling in an online organic chemistry preparation course relates to students' self-efficacy. Journal of Computing in Higher Education, 32(3), 505-528. https://doi.org/ 10.1007/s12528-019-09244-9

Zusho, A, Pintrich, P. R. \& Coppola, B. (2003). Skill and will: The role of motivation and cognition in the learning of college chemistry. International Journal of Science Education, 25(9), 1081-1094. https:/ / doi.org/10.1080/0950069032000052207 


\section{APPENDIX A}

\section{Experiment: Synthesis of Benzyl Ethyl Methyl Carbinol (=(2-Methyl-2-Hydroxy) Butylbenzene)}

\section{Warning}

Benzyl chloride is poisonous; it also dissolves simple surgical gloves!! The organometallic Grignard compound reacts violently with water, so use dry solvents and glassware in the first part of the procedure!!

\section{Procedure}

Use a $250 \mathrm{~mL}$ three-necked round-bottom flask provided with a stirrer (stirring bar), a dropping funnel and a reflux condenser. All openings to the air have to be closed with a stopper or fitted with a calcium chloride tube. Bring $2.1 \mathrm{~g}$ of magnesium shavings in the flask and a very small crystal of iodine. Prepare a solution of $10 \mathrm{~g}$ dry benzyl chloride in $60 \mathrm{~mL}$ of dry ether and transfer it into the dropping funnel. Add about $5 \mathrm{~mL}$ of this solution to the magnesium shavings and stir until the reaction starts. This is indicated by the disappearance of the brown color of the iodine, the appearance of some grey-white precipitate and the evolution of heat which causes the ether to reflux*. When the initial reaction slows down add the remaining ether solution at such a rate that the solution keeps refluxing gently. When the reaction is too vigorous the flask can be cooled by immersing in cold water. After the addition of all benzyl chloride reflux the solution for another 45 minutes. Usually the Grignard mixture now looks dark-gray.

Prepare a solution of $6 \mathrm{~g}$ butanone in $15 \mathrm{~mL}$ of dry ether and transfer it to the cleaned and dry dropping funnel. Add this solution dropwise to the Grignard mixture. A vigorous reaction occurs and the ether starts to reflux. External cooling may be necessary. After the addition the mixture is left stirring for 30 minutes. Meanwhile, prepare $80 \mathrm{~mL}$ of a $1 \mathrm{M}$ sulphuric acid solution. This is added dropwise to the reaction mixture. Again the reaction is vigourous, especially for the first few $\mathrm{mL}$. The resulting two phase system becomes clear after some stirring and is transferred to a separation funnel. Separate the organic layer and wash the aqueous layer two times with $20 \mathrm{~mL}$ of ether. Combine the organic layers and wash them twice with $20 \mathrm{~mL}$ of water. Dry with sodium sulphate and remove the ether with the rotatory evaporator. The crude benzylethylmethylcarbinol is purified by vacuum distillation (bp ca $115{ }^{\circ} \mathrm{C} / 15 \mathrm{mmHg}$ ). The first few mL's should be collected separately as they may contain impurities.

-Determine the yield and the boiling point of the product.

\section{Questions}

-Make an 1H NMR spectrum of the product (dissolve one drop in 0.5-0.8 $\mathrm{mL} \mathrm{CDCl3}$ ).

Give the reaction of the Grignard reagent with water.

Why can't ethyl acetate be used as solvent for the reaction? 


\section{APPENDIX B}

Questionnaire for Organic Chemistry Laboratory

Pretest Version

Please indicate the extent to which you agree with each of the following statements by filling the relevant choice.

\begin{tabular}{|c|c|c|c|c|c|c|}
\hline \# & Statements & 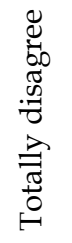 & 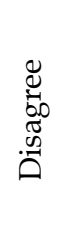 & 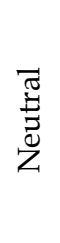 & 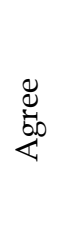 & 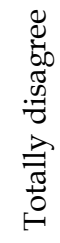 \\
\hline 1 & I expect that the organic chemistry lab will be fun. & (1) & (2) & (3) & (4) & (5) \\
\hline 2 & I expect to find the organic chemistry lab interesting. & (1) & (2) & (3) & (4) & (5) \\
\hline 3 & I expect to look forward to going to the organic chemistry lab. & (1) & (2) & (3) & (4) & (5) \\
\hline 4 & I expect that the organic chemistry lab will be dull. & (1) & (2) & (3) & (4) & (5) \\
\hline 5 & I expect to feel nervous in the organic chemistry lab. & (1) & (2) & (3) & (4) & (5) \\
\hline 6 & I expect walking into the organic chemistry lab will make me anxious. & (1) & (2) & (3) & (4) & (5) \\
\hline 7 & I expect the organic chemistry lab will make me feel uncomfortable and nervous. & (1) & (2) & (3) & (4) & (5) \\
\hline 8 & I expect the organic chemistry lab will make me feel uneasy and confused. & (1) & (2) & (3) & (4) & (5) \\
\hline 9 & I expect that I will be anxious about having to do the organic chemistry practicum & (1) & (2) & (3) & (4) & (5) \\
\hline 10 & I expect that I will be capable of obtaining good grades in the organic chemistry lab. & (1) & (2) & (3) & (4) & (5) \\
\hline 11 & I expect that I will be proud of my ability in the organic chemistry lab. & (1) & (2) & (3) & (4) & (5) \\
\hline 12 & I expect to feel good about my work in the organic chemistry lab. & (1) & (2) & (3) & (4) & (5) \\
\hline 13 & I expect to feel proud of my achievements in the organic chemistry lab. & (1) & (2) & (3) & (4) & (5) \\
\hline 14 & I expect to feel as good as the other people in the organic chemistry lab. & (1) & (2) & (3) & (4) & (5) \\
\hline 15 & $\begin{array}{l}\text { I expect to be able to handle even the most difficult tasks in the organic chemistry } \\
\text { practicum, if I have enough time. }\end{array}$ & (1) & (2) & (3) & (4) & (5) \\
\hline 16 & I expect to learn a lot from the organic chemistry lab. & (1) & (2) & (3) & (4) & (5) \\
\hline 17 & I expect to be able to handle more difficult tasks in the organic chemistry lab. & (1) & (2) & (3) & (4) & (5) \\
\hline 18 & I expect to perform well during the organic chemistry lab. & (1) & (2) & (3) & (4) & (5) \\
\hline 19 & I expect to be able to handle advanced work in the organic chemistry lab. & (1) & (2) & (3) & (4) & (5) \\
\hline
\end{tabular}




\section{APPENDIX C}

\section{Questionnaire for Organic Chemistry Laboratory}

\section{Posttest Version}

Please indicate the extent to which you agree with each of the following statements by filling the relevant choice.

\begin{tabular}{|c|c|c|c|c|c|c|}
\hline \# & Statements & 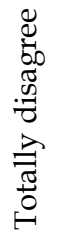 & 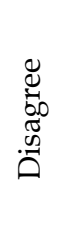 & $\begin{array}{l}\overline{\widetilde{Z}} \\
\stackrel{\Xi}{Z} \\
Z\end{array}$ & $\underset{\overleftarrow{b}}{\mathbb{Z}}$ & 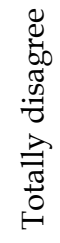 \\
\hline 1 & The organic chemistry lab is fun. & (1) & (2) & (3) & (4) & (5) \\
\hline 2 & I find the organic chemistry lab interesting. & (1) & (2) & (3) & (4) & (5) \\
\hline 3 & I actually look forward to going to the organic chemistry lab. & (1) & (2) & (3) & (4) & (5) \\
\hline 4 & The organic chemistry lab is dull. & (1) & (2) & (3) & (4) & (5) \\
\hline 5 & I feel nervous in the organic chemistry lab. & (1) & (2) & (3) & (4) & (5) \\
\hline 6 & Walking into the organic chemistry lab makes me anxious. & (1) & (2) & (3) & (4) & (5) \\
\hline 7 & The organic chemistry lab usually makes me feel uncomfortable and nervous. & (1) & (2) & (3) & (4) & (5) \\
\hline 8 & The organic chemistry lab makes me feel uneasy and confused. & (1) & (2) & (3) & (4) & (5) \\
\hline 9 & I dread having to do the organic chemistry lab. & (1) & (2) & (3) & (4) & (5) \\
\hline 10 & I am capable of obtaining good grades in the organic chemistry lab. & (1) & (2) & (3) & (4) & (5) \\
\hline 11 & I am proud of my ability in the organic chemistry lab. & (1) & (2) & (3) & (4) & (5) \\
\hline 12 & I feel good about my work in the organic chemistry lab. & (1) & (2) & (3) & (4) & (5) \\
\hline 13 & I am proud of my achievements in the organic chemistry lab. & (1) & (2) & (3) & (4) & (5) \\
\hline 14 & I am feeling as good as the other people in the organic chemistry lab. & (1) & (2) & (3) & (4) & (5) \\
\hline 15 & If I have enough time, I can do even the hardest tasks in the organic chemistry lab. & (1) & (2) & (3) & (4) & (5) \\
\hline 16 & I am sure that I can learn a lot in the organic chemistry lab. & (1) & (2) & (3) & (4) & (5) \\
\hline 17 & I think I can handle more difficult tasks in the organic chemistry lab. & (1) & (2) & (3) & (4) & (5) \\
\hline 18 & I know I can do well in the organic chemistry lab. & (1) & (2) & (3) & (4) & (5) \\
\hline 19 & I am sure I could do advanced work in the organic chemistry lab. & (1) & (2) & (3) & (4) & (5) \\
\hline
\end{tabular}




\section{APPENDIX D}

\section{Interviews with the Students Before the Course}

\section{Expectations}

What is your background?

What courses have you had that you found enjoyable? Why those courses?

Do you have any experience with organic chemistry? And practical organic chemistry?

What have you heard about this course from other students?

What are your expectations of the course? Do you think it will be fun?

What is your expectation of your own skills in an organic chemistry lab?

What do you think you will learn from the lab?

Do you think you will perform well?

VR

Do you think you can be proud of your work after this practical?

What are your views on VR?

\section{Motivation}

What do you think you will learn from the VR experience?

On a scale of 1 to 10 , how motivated are you for this practical?

Do you think VR will impact your motivation?

Do you think you will learn more from the VR experiences than a day in the lab without any prior training?

\section{Interviews with the Students Following the Course}

\section{Expectations}

What courses have you had that you found enjoyable?

What have you heard about this course from other students?

Do you think those statements were true?

What did you think of the organic chemistry lab?

What have you learned about your own skills?

What do you think you have learned?

Are you proud of the work you have done?

Did it meet your expectations?

Were you excited for the course?

VR

What did you think about the VR?

Was the VR easy to use?

Did it help you learn better?

\section{Laboratory Anxiety}

Is laboratory anxiety something you relate to?

Do you think it affected your motivation during the practical?

Did the VR help you deal with this?

Do you think VR might help with the anxiety?

\section{https://www.ejmste.com}

